\title{
Pesquisa qualitativa na pandemia da COVID-19: Uma experiência com homens e o tema da violência doméstica
}

\author{
Wanderlei Abadio de Oliveira ${ }^{1}$, Juliana Cristina Magrin ${ }^{1}$ e Julia Maria \\ Terossi Carvalho'
}

\author{
1 Programa de Pós-Graduação em Psicologia Pontifícia Universidade Católica de Campinas, \\ Brasil | wanderleio@hotmail.com; juliana_magrin@hotmail.com; \\ juliacarvalho.psico@gmail.com | https://orcid.org/0000-0002-3146-8197; \\ https://orcid.org/0000-0003-0169-7463; https://orcid.org/0000-0003-4734-7581
}

Resumo: Introdução. A pandemia da COVID-19 impactou a área da saúde, diferentes setores da sociedade, assim como a saúde mental das pessoas e o desenvolvimento de pesquisas com delineamentos diversos. Objetivos. Esse estudo teve como objetivo refletir sobre as dificuldades colocadas à pesquisa qualitativa em tempos de pandemia e o modo de as ultrapassar a partir de um relato de experiência. Métodos. Trata-se de um estudo descritivo, qualitativo, do tipo relato de experiência. $\mathrm{Na}$ análise da experiência foi utilizada a técnica expressivo-criativa "redemoinho de ideias". Resultados. A experiência foi exitosa, principalmente, no que se refere ao recrutamento de público-alvo para a pesquisa qualitativa. $\mathrm{Na}$ prática científica experienciada e relatada todos os pontos fortes de entrevistas on-line empregadas em pesquisas científicas foram alcançados: maior abrangência geográfica dos participantes; economia de recursos financeiros e redução de tempo na coleta de dados, maior segurança sanitária para participantes e pesquisadores e a possibilidade de investigar tópicos socialmente sensíveis (como a violência doméstica ou o ser violento) sem grandes constrangimentos. $\mathrm{Na}$ análise da experiência, verificou-se que a vivência da pandemia no campo da individualidade mobilizou sentimentos negativos e a perspectiva de que a pandemia seria situação temporária, mas sem potencial para afetar a pesquisa proposta. As respostas institucionais favoreceram a superação da crise e o surgimento de alternativas metodológicas. A resolução para os problemas enfrentados pelos pesquisadores passou por um processo heurístico de inventar novas formas de acessar fatos ou os fenômenos de interesse. Conclusões. Espera-se contribuir para a reflexão sobre as possibilidades do uso da tecnologia a partir de um caso específico, além de ampliar a discussão sobre a importância do detalhamento de métodos, instrumentos e procedimentos de coleta de dados em futuros estudos qualitativos desenvolvidos on-line.

Palavras-chave: Pandemia; Pesquisa Qualitativa; Violência.

Qualitative Research in the COVID-19 Pandemic: An Experience with Men and the Theme of Domestic Violence

\begin{abstract}
Introduction. The COVID-19 pandemic impacted the health area, different sectors of society, as well as people's mental health and the development of research with different designs. Goals. This study aimed to reflect on the difficulties posed to qualitative research in times of pandemic and how to overcome them based on an experience report. Methods. This is a descriptive, qualitative, experience report type study. In the analysis of the experience, the expressive-creative technique "swirl of ideas" was used. Results. The experience was successful, especially with regard to the recruitment of target audiences for qualitative research. In the scientific practice experienced and reported, all the strengths of online interviews used in scientific research were achieved: greater geographical coverage of the participants; savings in financial resources and reduction of time in data collection, greater health security for participants and researchers and the possibility of investigating socially sensitive topics (such as domestic violence or being violent) without major constraints. In the analysis of the experience, it was found that the experience of the pandemic in the field of individuality mobilized negative feelings and the perspective that the pandemic would be a temporary situation, but without the potential to affect the proposed research. Institutional responses favored overcoming the crisis and the emergence of methodological alternatives. The resolution to the problems faced by the researchers went through a heuristic process of inventing new ways of accessing facts or phenomena of interest. Conclusions. It is expected to contribute to the reflection on the possibilities of using technology from a specific case, in addition to broadening the discussion on the importance of detailing methods, instruments and procedures for data collection in future qualitative studies developed online.
\end{abstract}

Keywords: Pandemic; Qualitative Research; Violence. 


\section{Introdução}

A pandemia da COVID-19, doença causada pelo vírus SARS-CoV-2, já causou a infecção de mais de 127 milhões de pessoas no mundo e desse total mais de dois milhões foram vítimas fatais (dados de março de 2021, Painel Interativo - Coronavírus Brasil). Trata-se da maior crise sanitária e epidemiológica do século XXI (Oleribe et al., 2020). No mundo, todos os serviços de saúde foram reorganizados e momentos críticos de colapso desses serviços foram experimentados. Para conter o avanço do contágio pelo novo coronavírus medidas não farmacológicas foram recomendadas e implementadas em diferentes países, incluindo quarentenas, isolamento, restrições de mobilidade individual/interação face a face (aglomerações, distanciamento social), adoção de home office em áreas não essenciais, fechamento de escolas e universidades, entre outras medidas (Oliveira, Oliveira-Cardoso, Silva, \& Santos, 2020a).

Assim, percebe-se que os impactos desse momento pandêmico ultrapassam a dimensão dicotômica saúde-doença, pois eles não se referem apenas ao adoecimento ou fatalidade da doença, reforçando a compreensão ampliada de saúde como completo estado de bemestar e qualidade de vida. Em momentos de pandemia a vivência de problemáticas que ultrapassam o campo médico é comumente documentada na literatura científica. Uma revisão sistemática que incluiu 15 estudos verificou que comprometimento da saúde mental e diferentes manifestações de sofrimento psicológico é um dos impactos psicossociais mais referidos ou relacionados a esses momentos (Chu, Alam, Larson, \& Lin, 2020). Além disso, há um incremento das desigualdades sociais, ampliação da insegurança alimentar de muitos povos, desafios econômicos, acesso reduzido a cuidados de saúde, oferta alternativa de educação e violência doméstica. Essas consequências negativas foram verificadas em seis epidemias de doenças infecciosas, incluindo a atual pandemia da COVID- 19 (Chu et al., 2020).

No caso da violência doméstica, esse não é um problema novo, resultado colateral da pandemia. Contudo, estudos tem analisado como o isolamento social ou a quarentena, aplicados em muitos países, aumentou o contatado das mulheres com parceiros agressivos (Oliveira et al., 2020b; Sánchez, Vale, Rodrigues, \& Surita, 2020). Embora o acesso a dados concretos seja limitado, após adotarem as medidas de isolamento social ou quarentena, países como França e Argentina verificaram um aumento de cerca de 30\% e $25 \%$ nos casos de violência doméstica (Sri, Das, Gnanapragasam, \& Persaud, 2021). No Chipre e em Cingapura, serviços de atendimento às vítimas registraram um aumento nas ligações de $30 \%$ e $33 \%$, respectivamente (Sri et al., 2021). Em outros países, como Canadá, Alemanha, Espanha, Reino Unido, Estados Unidos da América e Brasil também avaliam aumento nos casos de violência doméstica contra mulheres (Oliveira et al., 2020b; Sri et al., 2021).

Além desse cenário, percebe-se que a pandemia afetou também o desenvolvimento das pesquisas científicas, principalmente as pesquisas qualitativas que precisaram se reinventar diante das limitações impostas pela própria doença e pela falta de acesso a potenciais participantes ou campos de coleta de dados. É sabido que alternativas no modo como as pesquisas são desenvolvidas já estavam sendo implementadas por pesquisadores em todo mundo, mas a pandemia acelerou o processo e incluiu radicalmente pesquisadores que não estavam preparados para lidar com as adversidades e as novas experiências tecnológicas, éticas e de adaptações metodológicas necessárias. Infere-se que muitos pesquisadores experimentaram sentimentos de frustração e desamparo, aspecto que salienta a importância de se registrar como diferentes pesquisas foram transformadas a partir da pandemia da COVID-19, como se propõe nesse trabalho.

\subsection{O Presente Estudo}

O objetivo deste estudo foi refletir sobre as dificuldades colocadas à pesquisa qualitativa em tempos de pandemia e o modo de as ultrapassar a partir de um relato de experiência. As questões norteadoras do trabalho foram: Como uma pesquisa qualitativa pôde ser desenvolvida durante a pandemia da COVID-19? 
Quais lições dessa experiência podem ser extraídas por outros pesquisadores a partir de um exemplo - relato de experiência de pesquisa com homens durante a pandemia?

\section{Metodologia}

Este é um estudo descritivo, qualitativo, do tipo relato de experiência. O estudo surgiu a partir da verificação empírica sobre os desafios enfrentados por diferentes pesquisadores no desenvolvimento de investigações qualitativas. O contexto da pandemia da COVID-19 provocou, principalmente, adaptações e a migração dos estudos qualitativos também para as ambiências da pesquisa digital, mas não se trata apenas de uma mudança no modo como se coleta os dados e é preciso ultrapassar as soluções estratégicas emergenciais geradas pelas contingências do momento (Deslandes \& Coutinho, 2020). Essa reflexão é importante, pois os estudos qualitativos não se valem apenas da coleta de dados em questionários estruturados, que inclusive são os modelos mais adotados atualmente para pesquisas em diferentes campos do saber. Assim, julgou-se pertinente a descrição de uma experiência que integra conhecimentos teóricos e práticos sobre como estão sendo pensadas ou implementadas essas pesquisas.

Originalmente, a pesquisa relatada previa um contato extenso entre a pesquisadora principal (JCM) e os pesquisados que seriam recrutados em serviços da justiça restaurativa destinados a homens acusados de praticar agressões contra suas companheiras. Nesse contato seriam realizadas entrevistas semiestruturadas. Contudo, logo após definição do público-alvo e serviços possíveis para o desenvolvimento da pesquisa, foi deflagrada a pandemia da COVID-19 e foram impossibilitados contatos presenciais com os serviços ou mesmo com o público específico (homens acusados de praticar violência doméstica). Após diversas sessões de orientação, decidiu-se por continuar com o projeto por meio de plataformas digitais e essa mudança exigiu o estabelecimento de novos objetivos e perfil dos participantes. A experiência corporificada da prática da violência doméstica foi abandonada e o estudo se voltou para os sentidos sobre esse fenômeno atribuídos por homens na população geral. Nesse ponto os pesquisadores se depararam com outro desafio: como selecionar participantes sem a possibilidade de mobilidade e as medidas de isolamento em curso, considerando possíveis vieses relacionados à seleção dos participantes? Optou-se, então, pela aplicação de um questionário on-line sobre a opinião de homens sobre o fenômeno em análise. Os homens foram convidados via redes socais e também foram disparados convites em grupos aleatórios de um aplicativo de troca de mensagens. As chamadas-convites para adesão foram disseminadas pelos pesquisadores envolvidos na proposta. Após responder ao questionário exploratório, os homens eram convidados para a segunda etapa da pesquisa que consistia em uma entrevista semiestruturada por videoconferência. Caso aderissem a esse convite, eles deixavam seus endereços de e-mail e a pesquisadora responsável (JCM) entrava em contato para agendar as entrevistas. Nessa etapa, foram excluídos potenciais participantes com vínculos com os pesquisadores para minimizar potenciais vieses de seleção. As entrevistas foram gravadas em áudio, com o consentimento dos participantes, e transcritas na íntegra.

$\mathrm{Na}$ análise da experiência foi utilizada a técnica expressivo-criativa "redemoinho de ideias". Trata-se de uma metáfora baseada em uma proposta pedagógica de ensinoaprendizagem (Ontoria, Gómez, \& Luque, 2014). No caso desse estudo, verificou-se ser importante considerar, para além da pesquisa qualitativa em si, os desafios e o modo como os pesquisadores significaram ou vivenciaram esse momento.

A prática científica experienciada e relatada em tela foi aprovada pelo Comitê de Ética em Pesquisa da Pontifícia Universidade Católica de Campinas (Parecer № 4.578.553). 


\section{Resultados e Discussão}

Mesmo com a gravidade da pandemia da COVID-19, pesquisadores qualitativos não perderam a esperança de desenvolver e executar investigações bem-sucedidas. Contudo, a realidade objetiva demandou a realização de ações específicas para mudar rotas previstas e evitar cancelamentos, além do confronto com a diminuição da dedicação ou motivação para o desenvolvimento dos estudos. No caso da pesquisa de fundo desse relato de experiência, desde o início, foram exploradas alternativas para o modelo tradicional adotado em pesquisas qualitativas que pressupõem o contato face to face com potenciais colaboradores. Na medida que a pandemia avançou pelo ano de 2020, a compreensão sobre os procedimentos a serem adotados pela equipe foi sendo revista e um modelo substitutivo foi constituído.

Vale ressaltar que o cenário da pandemia provocou atraso no desenvolvimento de ferramentas diagnósticas, no cuidado oferecido a diferentes populações, no progresso de pesquisas com diferentes desenhos qualitativos e, além disso, um processo de desistência de pesquisadores com vínculos precários ou em início de carreira na medida em que afetam severamente as condições de vida que já foram comprometidas pela pandemia (Osório, 2020; Simmons \& Luck, 2020). Assim, esse trabalho é uma iniciativa para demonstrar como limitações e aspectos individuais foram sendo superados, bem como a pesquisa qualitativa foi se transformando e não interrompida mesmo com as adversidades que se colocaram para os pesquisadores.

Nesse sentido, é possível assinalar o sucesso da mudança de estratégia para recrutar os participantes, talvez a mudança mais radical no processo investigativo, a partir da divulgação e aplicação de um questionário on-line. No total, 164 homens responderam ao instrumento composto por 26 perguntas divididas em dois blocos: caracterização socioeconômica e opinião sobre violência doméstica contra a mulher. Sobre o perfil dos respondentes, observa-se que $56 \%$ possuíam de 18 a 24 anos, mais de $75 \%$ se autodeclaram brancos e heterossexuais. A maioria dos participantes $(59 \%)$ também possuíam ensino superior. Nove estados brasileiros foram representados pelos respondentes, mas a maioria morava no estado de São Paulo. Para etapa qualitativa, 39 homens se voluntariaram.

Nas entrevistas, também realizadas remotamente, percebe-se que os homens têm uma necessidade excessiva de falar sobre suas vivências, principalmente tentando explicar ou justificar algumas questões abordadas no questionário exploratório (sobre quem pode praticar violência doméstica contra a mulher; o que pensam/sentem pelos agressores ou pelas vítimas quando tem acesso a uma notícia de feminicídio, por exemplo). No geral, nesses encontros virtuais também é possível perceber, preliminarmente, um entendimento mais crítico sobre a gravidade do fenômeno investigado e a necessidade de se construir políticas públicas de defesa das mulheres. Também se verificou grande interesse dos participantes pela temática da pesquisa. Não foram identificados dificuldades ou entraves dos participantes no que se refere ao modelo de coleta de dados adotado - entrevistas por videoconferência gravadas.

Nota-se que as entrevistas realizadas por videoconferência ou on-line (via diferentes recursos) não começaram a ser implementadas a partir da pandemia, sendo considerada um procedimento de pesquisa qualitativa sério e viável (Gonçalo \& Barros, 2014; Nicolacida-Costa, Romão-Dias \& Di Luccio, 2009). Segundo a literatura científica, as entrevistas on-line são adequadas sempre que os participantes do estudo estejam familiarizados com o uso das ferramentas adotadas e o objetivo da pesquisa permita sua implementação. $O$ grande problema que se coloca para esse modelo é a impossibilidade de se recrutar online perfis semelhantes ou de interesse específico (Nicolaci-da-Costa, Romão-Dias \& Di Luccio, 2009), por isso nessa pesquisa se ampliou o perfil necessário para adesão ao estudo em sua etapa qualitativa. 
Ainda vale ressaltar que o modo como as pesquisas que utilizam os recursos on-line no processo de desenvolvimento, não esclarecem, muitas vezes, como os procedimentos metodológicos foram planejados ou conduzidos e as principais justificativas utilizadas para o emprego do modelo on-line (Gonçalo \& Barros, 2014). Esse é um tema que necessita ser debatido na produção do conhecimento e esse trabalho contribuí nessa direção.

Além disso, conforme sistematizado por Schmidt, Palazzi e Piccinini (2020), a prática científica experienciada e relatada, todos pontos fortes de entrevistas on-line empregadas em pesquisas científicas. Com o recrutamento também on-line, nesse sentido, se garantiu uma maior abrangência geográfica - aspecto importante considerando o tamanho continental de países como o Brasil. Houve ainda economia de recursos financeiros e redução de tempo na coleta de dados, maior segurança sanitária para participantes e pesquisadores e a possibilidade de investigar tópicos socialmente sensíveis (como a violência doméstica ou o ser violento) sem constrangimentos pois o contato face to face está sendo mediado pela tecnologia.

\subsection{Síntese Interpretativa: a Metáfora do Redemoinho}

Esse estudo objetivou apresentar uma experiência de pesquisa qualitativa com homens sobre violência doméstica durante a pandemia da COVID-19. Nesse sentido, os participantes foram recrutados por meio da aplicação de um questionário exploratório divulgado em plataformas de mídia social. Esse não era o modelo proposto originalmente pelos pesquisadores, mas sim um modelo alternativo que emergiu após a eclosão da pandemia da COVID-19. Assim, a experiência dos pesquisadores foi interpretada segundo a técnica expressivo-criativa "redemoinho de ideias" (Ontoria et al., 2014). As transformações metodológicas provocaram sentimentos, impressões e novos significados (os pesquisadores no centro) sobre como conduzir a pesquisa qualitativa diante de uma situação percebida como sem controle (o redemoinho; a pandemia). Nesse sentido, evidenciou-se dois níveis de complexidade. No primeiro nível aparece o impacto da pandemia na vivência prática dos pesquisadores, incluindo os sentimentos envolvidos. No outro nível foram reveladas as respostas institucionais de suporte, o aumento do tempo de trabalho em tela e o aumento do número de pesquisas divulgadas on-line por diferentes grupos de pesquisas, com diferentes interesses e delineamentos. Segundo esse modelo interpretativo, o desenvolvimento da pesquisa qualitativa e os impactos do modelo remoto no desenvolvimento e na qualidade dos dados estão no centro. Essas interpretações estão sintetizadas e representadas na Figura 1.

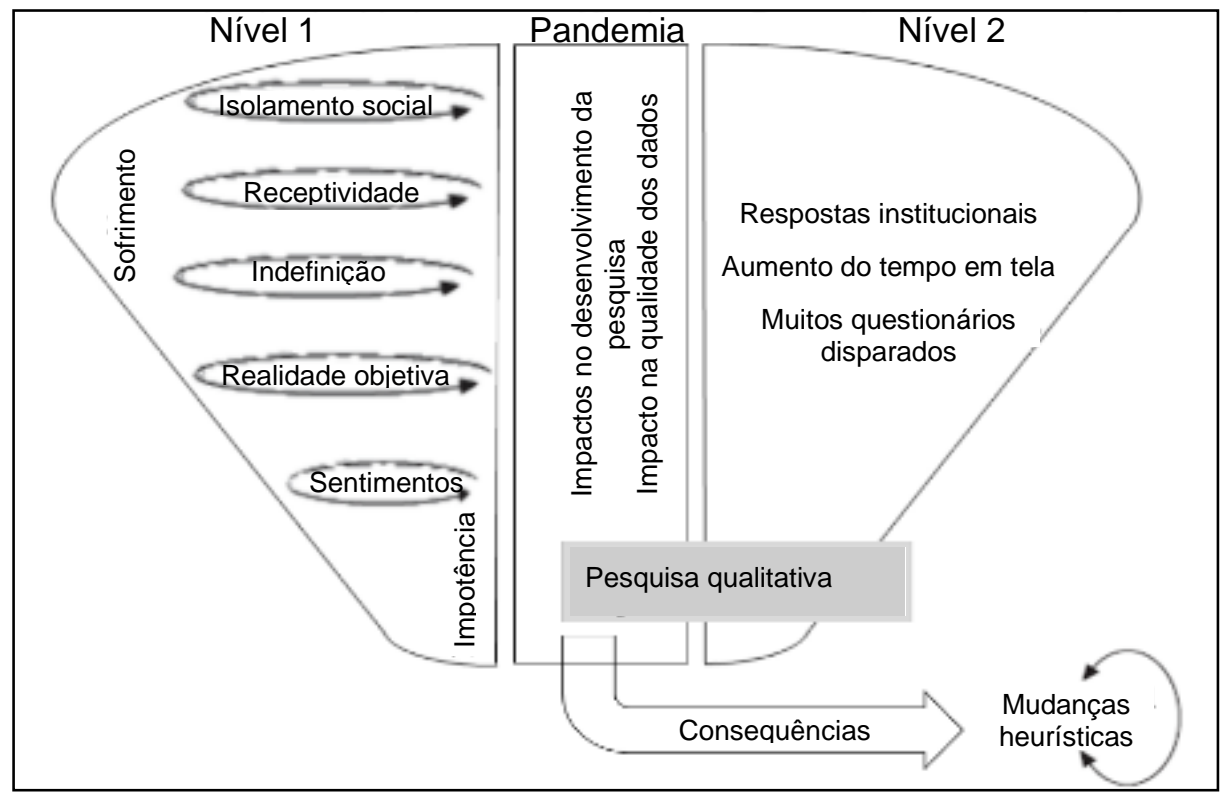

Fig. 1 Interpretação segundo a metáfora do redemoinho. 
No primeiro momento se observa a vivência da pandemia no campo da individualidade, sendo mobilizados sentimentos negativos e a perspectiva de que a pandemia seria situação temporária, mas sem potencial para afetar a pesquisa proposta. É a partir das respostas institucionais que os sentimentos individuais negativos, de impotência e desamparo, por exemplo, vão sendo superados e alternativas metodológicas vão sendo delineadas. Há, ativamente, uma elaboração cognitiva do momento vivido, considerando que os impasses e questionamentos apresentados necessitavam de resolução estrutural e essa resolução passou por um processo heurístico de inventar novas formas de acessar fatos ou os fenômenos de interesse dos pesquisadores.

\section{Conclusões}

Esse trabalho apresentou os recursos metodológicos, questionário on-line e entrevista por videoconferência, como elementos chave da coleta de dados de uma pesquisa qualitativa durante a pandemia da COVID-19. A experiência foi exitosa, principalmente, no recrutamento de público-alvo para uma pesquisa qualitativa. A divulgação em redes sociais e aplicativos para troca de mensagens se mostrou como estratégia válida na difusão do estudo fornecendo links para acesso ao questionário exploratório sobre as questões de pesquisa. Essas plataformas de mídia social parecem ser ferramentas poderosas para manter os pesquisadores qualitativos conectados à população em geral ou públicos específicos durante o momento pandêmico. Espera-se contribuir para a reflexão sobre as possibilidades do uso da tecnologia em um caso específico, além de ampliar a discussão sobre a importância do detalhamento de métodos, instrumentos e procedimentos de coleta de dados em futuros estudos qualitativos.

\section{Agradecimentos}

Os autores agradecem ao CNPq (Brasil) pela concessão de Bolsa Produtividade para o terceiro autor e bolsa de mestrado para a segunda autora.

\section{Referências}

Chu, I. Y., Alam, P., Larson, H. J., \& Lin, L. (2020). Social consequences of mass quarantine during epidemics: a systematic review with implications for the COVID-19 response. Journal of Travel Medicine, 27(7), taaa192. https://doi.org/10.1093/jtm/taaa192

Deslandes, S., \& Coutinho, T. (2020). Pesquisa social em ambientes digitais em tempos de COVID-19: notas teórico-metodológicas. Cadernos de Saúde Pública, 36(11), e00223120. https://dx.doi.org/10.1590/0102-311x00223120

Gonçalo, C. S., \& Barros, N. F. (2014). Entrevistas telefônicas na pesquisa qualitativa em saúde. Saúde \& Transformação Social, 5(1), 22-26. http://pepsic.bvsalud.org/scielo.php?script=sci_arttext\&pid=S217870852014000100005\&lng=pt\&tlng=pt.

Nicolaci-da-Costa, A. M., Romão-Dias, D., \& Di Luccio, F. (2009). Uso de entrevistas on-line no método de explicitação do discurso subjacente (MEDS). Psicologia: Reflexão e Crítica, 22(1), 36-43. https://doi.org/10.1590/S0102-79722009000100006

Oleribe, O., Ezechi, O., Osita-Oleribe, P., Olawepo, O., Musa, A. Z., Omoluabi, A. . . TaylorRobinson, S. D. (2020). Public perception of COVID-19 management and response in Nigeria: a cross-sectional survey. BMJ open, 10(10), e041936. https://doi.org/10.1136/bmjopen-2020-041936

Oliveira, W. A., Magrin, J. C., Andrade, A. L. M., Micheli, D., Carlos, D. M., Fernandez, J. E. R., Silva, M. A. I., \& Santos, M. A. (2020a). Violência por parceiro íntimo em tempos da COVID19: scoping review. Psicologia, Saúde \& Doenças. 21(3), 606-623.

http://dx.doi.org/10.15309/20psd210306 
Oliveira, W. A., Oliveira-Cardoso, É. A., Silva, J. L., \& Santos, M. A. (2020b). Impactos psicológicos e ocupacionais das sucessivas ondas recentes de pandemias em profissionais da saúde: revisão integrativa e lições aprendidas. Estudos de Psicologia (Campinas), 37, e200066. https://doi.org/10.1590/1982-0275202037e200066

Ontoria, A., Gómez, J. P. R., \& Luque, A. (2014). Aprendizaje centrado en el alumno (3 ed.). Madrid: Narcea.

Sánchez, O. R., Vale, D. B., Rodrigues, L., \& Surita, F. G. (2020). Violence against women during the COVID-19 pandemic: An integrative review. International journal of gynaecology and obstetrics, 151(2), 180-187. https://doi.org/10.1002/ijgo.13365

Schmidt, B, Palazzi, A., Piccinini, C. A. (2020). Online interviews: potential and challenges for data collection in the context of the COVID-19 pandemic. REFACS, 8(4), 960-966. https://doi.org/10.18554/refacs.v8i4.4877

Sri, A. S., Das, P., Gnanapragasam, S., \& Persaud, A. (2021). COVID-19 and the violence against women and girls: 'The shadow pandemic.' International Journal of Social Psychiatry. https://doi.org/10.1177/0020764021995556 